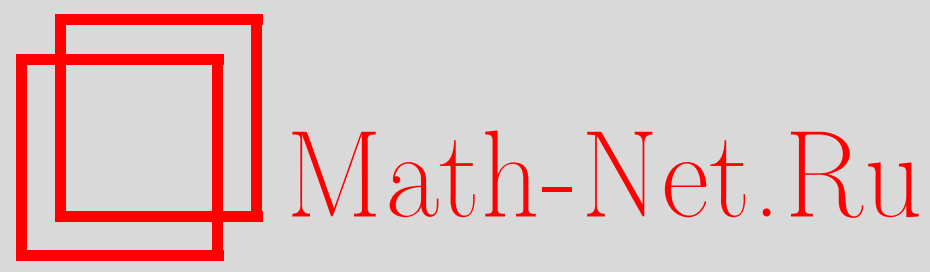

И. Х. Мусин, Сюръективность линейного дифференциального оператора в весовом пространстве бесконечно дифференцируемых функций, Матем. заметки, 2002, том 71, выпуск $5,713-724$

DOI: https://doi.org/10.4213/mzm379

Использование Общероссийского математического портала Math-Net.Ru подразумевает, что вы прочитали и согласны с пользовательским соглашением http://www.mathnet.ru/rus/agreement

Параметры загрузки:

IP : 54.166 .219 .16

26 апреля 2023 г., 16:22:12

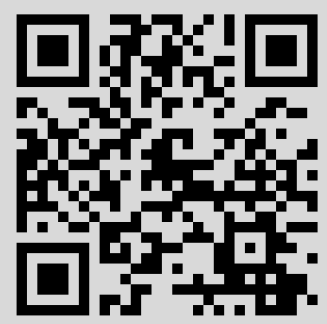




\section{СЮРЪЕКТИВНОСТЬ ЛИНЕЙНОГО ДИФФЕРЕНЦИАЛЬНОГО OПЕРАТОРА В ВЕСОВОМ ПРОСТРАНСТВЕ БЕСКОНЕЧНО ДИФФЕРЕНЦИРУЕМЫХ ФУНКЦИЙ}

И. Х. Мусин

Приводится достаточное условие сюръективности линейного дифференциального оператора бесконечного порядка с постоянными коэффициентами в весовом пространстве бесконечно дифференцируемых функций на вещественной прямой.

Библиография: 18 названий.

1. Введение. Обозначим через $V$ класс неотрицательных выпуклых возрастающих функций $v$ на $[0, \infty), v(0)=0$, удовлетворяющих условиям:

1) $\lim _{x \rightarrow+\infty} \frac{v(x+1)-v(x)}{x}=0$;

2) $\exists A_{v}, B_{v} \in \mathbb{R}: \forall x \geqslant 1 v(x) \geqslant x \ln x+A_{v} x+B_{v}$.

Зафиксируем $\alpha>1$. Пусть $\psi: \mathbb{R} \rightarrow[0, \infty)$ - выпуклая функция, удовлетворяющая условиям:

1) существует постоянная $A_{\psi}>0$ такая, что для любых $x_{1}, x_{2} \in \mathbb{R}$

$$
\left|\psi\left(x_{1}\right)-\psi\left(x_{2}\right)\right| \leqslant A_{\psi}\left(1+\left|x_{1}\right|+\left|x_{2}\right|\right)^{\alpha-1}\left|x_{1}-x_{2}\right|
$$

2) $\lim _{x \rightarrow \infty} \frac{\psi(x)}{|x|}=+\infty$.

Пусть $\varphi(x)=\sup _{y \in \mathbb{R}}(x y-\psi(y)), x \in \mathbb{R},-$ преобразование Юнга функции $\psi$. Положим $\theta_{m}(x)=\exp (\varphi(x)-m \ln (1+|x|)), x \in \mathbb{R}, m \in \mathbb{N}$. Пусть всюду далее $\left\{\varepsilon_{m}\right\}_{m=1}^{\infty}-$ строго убывающая к нулю последовательность положительных чисел, а $\sigma$ - положительное число. Как обычно, $\mathscr{E}(\mathbb{R})$ - пространство бесконечно дифференцируемьх функций на вещественной прямой, $H(\mathbb{C})$ - пространство целых функций.

Пусть $v \in V$. Для $m \in \mathbb{N}$ введем нормированные пространства

$$
G_{m}=\left\{f \in \mathscr{E}(\mathbb{R}): p_{m}(f)=\sup _{x \in \mathbb{R}, k \in \mathbb{Z}_{+}} \frac{\left|f^{(k)}(x)\right|}{\left(\sigma+\varepsilon_{m}\right)^{k} \exp (v(k)) \theta_{m}(x)}<\infty\right\} .
$$

Через $G$ обозначим проективный предел пространств $G_{m}$ относительно вложений $i_{m n}$ : $G_{m} \rightarrow G_{n}, m>n$.

Работа выполнена при финансовой поддержке Российского фонда фундаментальных исследований, грант № 99-01-00655. 
Напомним, что пространство, представимое в виде проективного предела последовательности нормированных пространств $S_{n}, n=1,2, \ldots$, относительно линейных непрерьвных отображений $g_{m n}: S_{n} \rightarrow S_{m}, m<n$, таких, что $g_{n, n+1}$ вполне непрерьвны для каждого $n$, назьвается пространством $\left(M^{*}\right)$ [1]. В работе [2] показано, что вложения $i_{m, m+1}: G_{m+1} \rightarrow G_{m}$ вполне непрерывны для каждого $m$. Поэтому $G$ - пространство $\left(M^{*}\right)$.

Очевидно, определение пространства $G$ не зависит от выбора конкретной последовательности $\left\{\varepsilon_{m}\right\}_{m=1}^{\infty}$. В случае вьполнения условия

$$
\varlimsup_{k \rightarrow \infty}\left(\frac{\exp (v(k))}{k !}\right)^{1 / k}<\infty
$$

функции из $G$ будут вещественно аналитичны.

Положим $w(r)=\sup _{k \in \mathbb{Z}_{+}}(k \ln r-v(k)), r>0, w(0)=0$. Функция $w$ непрерывна на неотрицательной полуоси $[3$, c. 24]. Очевидно, $w(|z|), z \in \mathbb{C},-$ субгармоническая функция в комплексной плоскости и $\ln r=o(w(r)), r \rightarrow+\infty$. Из того, что $w(r)=0$ для $r \in[0, \exp (v(1))]$, и из второго условия на $v$ следует, что найдется постоянная $C_{v}>0$ такая, что $w(r) \leqslant C_{v} r, r \geqslant 0$. Известно [2], [4], что для любых $z_{1}, z_{2} \in \mathbb{C}$

$$
\left|w\left(\left|z_{2}\right|\right)-w\left(\left|z_{1}\right|\right)\right| \leqslant C_{v} e\left|z_{2}-z_{1}\right| \text {. }
$$

Положим $w_{m}(|z|)=w\left(\left(\sigma+\varepsilon_{m}\right)^{-1}|z|\right), z \in \mathbb{C}, m \in \mathbb{N}$.

Через $P$ обозначим индуктивньй предел нормированных пространств

$$
P_{m}=\left\{f \in H(\mathbb{C}):\|f\|_{m}=\sup _{z \in \mathbb{C}} \frac{|f(z)|}{\exp \left(\psi(\operatorname{Im} z)+w_{m}(|z|)\right)}<\infty\right\}, \quad m \in \mathbb{N},
$$

относительно вложений $j_{m n}: P_{m} \rightarrow P_{n}, m<n$.

Как известно, пространство, представимое в виде внутреннего индуктивного предела последовательности нормированных пространств $S_{n}, n=1,2, \ldots$, каждое из которых вложено в последующее вполне непрерывно, назьвается пространством $\left(L N^{*}\right)[1]$. В работе [2] показано, что вложения $j_{n, n+1}: P_{n} \rightarrow P_{n+1}$ вполне непрерьвны. Поэтому $P$ - пространство $\left(L N^{*}\right)$.

Через $G^{*}$ обозначаем сильное сопряженное пространство к пространству $G$. Определим преобразование Фурье-Лапласа $\widehat{T}$ функционала $T \in G^{*}$ формулой $\widehat{T}(z)=T\left(e^{-i x z}\right)$, $z \in \mathbb{C}$.

В работе [2] получена

ТЕоремА А. Преобразование Фурье-Лапласа устанавливает топологический изоморфизм между пространствами $G^{*}$ и $P$.

В случае, когда сходится ряд $\sum_{k=0}^{\infty} \exp (v(k)-v(k+1))$, данная теорема установлена в $[5]$.

В данной работе изучается задача о разрешимости линейных дифференциальных уравнений бесконечного порядка с постоянными коэффициентами в пространстве $G$.

Всюду далее предполагается, что функция $v \in V$ удовлетворяет условиям:

V1. $\exists a>0, b \in \mathbb{R}: \forall x \geqslant 0 v(2 x) \geqslant 2 v(x)+a x+b$;

V2. $\exists \varepsilon_{0}>0: \forall \varepsilon \in\left(0, \varepsilon_{0}\right) \exists a_{\varepsilon}, b_{\varepsilon}>0: \forall y \geqslant 1$

$$
\sup _{x \geqslant 1}(v(x+y)-v(x)-\varepsilon x) \leqslant v(y)+a_{\varepsilon} y+b_{\varepsilon} .
$$


Пусть $L(z)=\sum_{k=0}^{\infty} d_{k} z^{k}$ - целая функция, удовлетворяющая условиям:

L1. $\forall \varepsilon>0 \exists C_{\varepsilon}>0: \forall z \in \mathbb{C}|L(z)| \leqslant C_{\varepsilon} \exp (\varepsilon w(|z|))$;

L2. для $z \in \mathbb{C}$ вне некоторого множества $S$ непересекающихся кружков $D\left(\lambda_{j}, r_{j}\right)=$ $\left\{z \in \mathbb{C}:\left|z-\lambda_{j}\right|<r_{j}\right\}, j=1,2, \ldots$, таких, что

S1. $\lim _{j \rightarrow \infty} \lambda_{j}=\infty$,

S2. $\lim _{j \rightarrow \infty} \frac{r_{j}\left(1+\left|\lambda_{j}\right|+r_{j}\right)^{\alpha-1}}{w\left(\left|\lambda_{j}\right|\right)}=0$,

при любом $\varepsilon>0$ имеет место неравенство

$$
|L(z)| \geqslant c_{\varepsilon} \exp (-\varepsilon w(|z|))
$$

где $c_{\varepsilon}>0$ - некоторая постоянная.

В этих предположениях относительно $v$ и $L$ справедлива

ТЕоремА. Для любого $g \in G$ уравнение $\sum_{k=0}^{\infty} d_{k} i^{k} f^{(k)}(x)=g(x), x \in \mathbb{R}$, разреиимо в $G$.

Проводится рассмотрение именно дифференциального оператора по существу, так как зазор между функциями $\ln \theta_{m}(x)$ логарифмический и

$$
\lim _{x \rightarrow+\infty} \frac{\varphi(x)}{x \ln x}=+\infty \text {. }
$$

Как известно (см., например, [6], [7]), сюръективность линейных дифференциальных операторов с постоянными коэффициентами, операторов свертки в различных классах бесконечно дифференцируемых функций или аналитических функций часто благодаря использованию преобразования Фурье-Лапласа и методов функционального анализа [8] эквивалентна проблеме деления [9] в подходяших пространствах целых функций. Этот прием стал известен благодаря работам Мальгранжа [10], который положил начало изучению уравнений в свертках, и Эренпрайса [9], который охарактеризовал сюръективные операторы свертки на $\mathscr{E}\left(\mathbb{R}^{n}\right)$ в терминах оценок снизу на характеристическую функцию оператора, и затем использовался во многих работах (см. обзор в [7]). Например, в работах [11]-[13] результаты Эренпрайса были распространены на операторы свертки на пространствах ультрадифференцируемых функций [14], [15].

Из результатов по сюръективности линейных дифференциальных операторов бесконечного порядка с постоянными коэффициентами в классах бесконечно дифференцируемых функций на вещественной прямой с весовыми оценками на производные любого порядка известны лишь два - Ю. Ф. Коробейника [16] и автора [4]. Однако в работе [16] изучалось пространство, существенно отличающееся по своей природе от пространства $G$. В [4] рассматривается подобное $G$ пространство, но на характеристическую функцию оператора были наложены слишком жесткие условия.

Задача, изучаемая в данной работе, также сводится к проблеме деления (лемма 6).

План работы таков. В пункте 2 приводятся достаточное условие на неотрицательную вьпуклую возрастающую функцию на $[0, \infty)$, обеспечивающее для этой функции выполнение условия V2, и примеры функций из класса V, для которых вьполнены условия V1, V2. В пункте 3 приводится пример целой функции, удовлетворяющей условиям L1, L2. Пункт 4 посвящен вспомогательным утверждениям, используемьм при доказательстве теоремы. Доказательство теоремы приводится в п. 5 . 
2. Один способ проверки выполнения условия V2. При проверкевыполнения условия V2 в конкретных ситуациях может быть полезна

ЛЕмма 1. Пусть $и:[0, \infty) \rightarrow[0, \infty)$ - выпуклая возрастающая дважды непрерывно дифферениируемая функиия такая, что

1) $\lim _{x \rightarrow+\infty} \frac{u(x+1)-u(x)}{x}=0$;

2) существует постоянная $C>0$ такая, что $u^{\prime \prime}(x) \leqslant C x^{-1} n p u x \geqslant 1$.

Тогда для любого $\varepsilon \in(0, C)$ найдется постоянная $Q_{\varepsilon}$ такая, что для любого у $\geqslant 1$

$$
\sup _{x \geqslant 1}(u(x+y)-u(x)-\varepsilon x)<u(y)+\left(C \ln \frac{2 C}{\varepsilon}+\frac{5 C}{4}\right) y+Q_{\varepsilon} .
$$

ДокаЗАТЕЛьство. Пусть $y \geqslant 1$. Тогда $y \in[N, N+1)$ для некоторого $N \in \mathbb{N}$. Пусть $\varepsilon \in(0, C)$. Оценим сверху $\sup _{x \geqslant 1}(u(x+y)-u(x)-\varepsilon x-u(y))$. Вначале, пользуясь первым условием на $u$, найдем постоянную $q_{\varepsilon}>0$ такую, что $u(x+1)<u(x)+\varepsilon x / 2+q_{\varepsilon}, x \geqslant 0$. Далее имеем

$$
\begin{aligned}
& \sup _{x \geqslant 1}(u(x+y)-u(x)-\varepsilon x-u(y)) \leqslant \sup _{x \geqslant 1}(u(x+N+1)-u(x)-\varepsilon x-u(N)) \\
& \leqslant \sup _{x \geqslant 1}\left(u(x+N)-u(x)-\frac{\varepsilon x}{2}-u(N+1)\right)+\varepsilon N+2 q_{\varepsilon} .
\end{aligned}
$$

Отметим, что

$$
\begin{gathered}
u(x+N)-u(x)=\sum_{k=1}^{N}(u(x+k)-u(x+k-1)) \leqslant \sum_{k=1}^{N} u^{\prime}(x+k), \\
u(N+1)=\sum_{k=1}^{N}(u(k+1)-u(k))+u(1)>\sum_{k=1}^{N} u^{\prime}(k) .
\end{gathered}
$$

Следовательно,

$$
\begin{aligned}
& u(x+N)-u(x)-u(N+1)<\sum_{k=1}^{N}\left(u^{\prime}(x+k)-u^{\prime}(k)\right) \\
& =\sum_{k=1}^{N} \int_{k}^{x+k} u^{\prime \prime}(t) d t \leqslant C \sum_{k=1}^{N} \int_{k}^{x+k} \frac{d t}{t}=C \sum_{k=1}^{N} \ln \left(1+\frac{x}{k}\right) .
\end{aligned}
$$

Таким образом,

$$
\begin{aligned}
& \sup _{x \geqslant 1}\left(u(x+N)-u(x)-u(N+1)-\frac{\varepsilon x}{2}\right) \leqslant \sup _{x \geqslant 1}\left(C \sum_{k=1}^{N} \ln \left(1+\frac{x}{k}\right)-\frac{\varepsilon x}{2}\right) \\
& \quad \leqslant \sum_{k=1}^{N} \sup _{x \geqslant 1}\left(C \ln \left(1+\frac{x}{k}\right)-\frac{\varepsilon x}{2 N}\right)=N C \ln \frac{2 C}{e \varepsilon}+C N \ln N-C \sum_{k=1}^{N} \ln k+\frac{\varepsilon(N+1)}{4} .
\end{aligned}
$$


И так как $\sum_{k=1}^{N} \ln k \geqslant \int_{1}^{N} \ln x d x=N \ln N-N+1$, то

$$
\sup _{x \geqslant 1}\left(u(x+N)-u(x)-u(N+1)-\frac{\varepsilon x}{2}\right) \leqslant N C \ln \frac{2 C}{\varepsilon}-C+\frac{\varepsilon(N+1)}{4} .
$$

Следовательно, для $y \geqslant 1$

$$
\sup _{x \geqslant 1}(u(x+y)-u(x)-\varepsilon x-u(y))<\left(C \ln \frac{2 C}{\varepsilon}+\frac{5 C}{4}\right) y-C+\frac{\varepsilon}{4}+2 q_{\varepsilon} .
$$

Отсюда следует утверждение леммы.

ЗАМЕЧАНИЕ. Отметим, что для любой возрастающей функции $u$, удовлетворяющей условию $\mathrm{V} 2, \lim _{x \rightarrow+\infty}(u(x+1)-u(x)) x^{-1}=0$.

Приведем примеры функций из класса $V$, удовлетворяюших условиям V1, V2.

1. $v_{1}(x)=\rho x \ln (x+1), \rho \geqslant 1, x \geqslant 0$. Проверка принадлежности $v_{1}$ к $V$ и условия V1 не вызывает затруднений. Так как

$$
v_{1}^{\prime \prime}(x)=\frac{\rho}{x+1}+\frac{\rho}{(x+1)^{2}},
$$

то условие V2 выполняется в силу леммы 1 . Отметим также, что в этом случае при $r>e^{\rho} \rho e^{-1} r^{1 / \rho}-2 \ln r \leqslant w(r) \leqslant \rho e^{-1} r^{1 / \rho}$.

2. $v_{2}(x)=\rho \ln \Gamma(x+2), \rho \geqslant 1, x \geqslant 0$, где $\Gamma(x)$ - гамма-функция Эйлера. Функция $v_{2}$ вьпуклая на $[0, \infty)[17$, c. 763$]$. Функция $v_{2}$ возрастает на $[0, \infty)$. Это следует из исследования свойств гамма-функции, проведенного в $[17$, с. 755$]$. Пользуясь формулой Стирлинга, легко проверить, что условие V1 вьполняется. Далее, поскольку $(\ln \Gamma(x+2))^{\prime \prime}=\sum_{k=2}^{\infty} 1 /(x+k)^{2}$ (см., например, [17, формула $(28)$, с. 774$]$, отсюда

$$
v_{2}^{\prime \prime}(x)<\rho \int_{1}^{\infty} \frac{d t}{(x+t)^{2}}=\frac{\rho}{x+1},
$$

и тем самым условие V2 тоже выполнено.

Незначительно изменив функцию $\rho \ln \Gamma(x+1), \rho \geqslant 1$, например, на отрезке [0,2], можно получить функцию из класса $V$, удовлетворяющую условиям $\mathrm{V} 1, \mathrm{~V} 2$.

3. $v_{3}(x)=(x+1) \ln (x+1) \operatorname{arctg}(x+1), x \geqslant 0$. Имеем $v_{3}(0)=0$,

$$
v_{3}^{\prime}(x)=(\ln (x+1)+1) \operatorname{arctg}(x+1)+\frac{(x+1) \ln (x+1)}{1+(x+1)^{2}}>0 \quad \text { при } x \geqslant 0 .
$$

Очевидно, функция $v_{3}$ возрастающая на $[0, \infty)$. Так как

$$
v_{3}^{\prime \prime}(x)=\frac{\operatorname{arctg}(x+1)}{x+1}+\frac{2 \ln (x+1)}{\left(1+(x+1)^{2}\right)^{2}}+\frac{2}{1+(x+1)^{2}},
$$

то $v_{3}$ вьпуклая функция на $[0, \infty)$, удовлетворяющая условию V2. Далее, для $x>0$

$$
\begin{aligned}
v_{3}(2 x)-2 v_{3}(x)= & 2 x \ln (2 x+1) \operatorname{arctg}(2 x+1)-2 x \ln (x+1) \operatorname{arctg}(x+1) \\
& +\ln (2 x+1) \operatorname{arctg}(2 x+1)-2 \ln (x+1) \operatorname{arctg}(x+1) \\
\geqslant & \frac{\pi x}{2} \ln \frac{3}{2}+\frac{\pi}{4} \ln (2 x+1)-\pi \ln (x+1) .
\end{aligned}
$$

Отсюда следует, что условие $\mathrm{V} 1$ для $v_{3}$ вьполняется. 
3. Пример целой функции, удовлетворяющей условиям L1 и L2. Пусть $w_{1}$ - неотрицательная субгармоническая функция такая, что $w_{1}(r)=o(w(r))$ при $r \rightarrow$ $+\infty$ и $\ln (1+r)=o\left(w_{1}(r)\right)$ при $r \rightarrow+\infty$. Функция $w_{1}$ может быть построена, например, как в работе [15, лемма 1.7]. По теореме 6 из [18] существует целая функция $Y$ с простьми нулями, удовлетворяющая условиям:

Y1. круги $D\left(\mu_{k}, d\right)=\left\{z \in \mathbb{C}:\left|z-\mu_{k}\right|<d\right\}$, где $d$-некоторое положительное число, $\mu_{1}, \mu_{2}, \ldots, \mu_{k}, \ldots$ - нули функции $Y$, расположенные в порядке неубывания их модулей, попарно не пересекаются;

Y2. вне множества $\bigcup_{k=1}^{\infty} D\left(\mu_{k}, d\right)$ при некоторых положительных $B, B_{0}$ вьполняется неравенство

$$
\left|w_{1}(|z|)-\ln \right| Y(z)|| \leqslant B \ln (1+|z|)+B_{0} .
$$

Без ограничения общности считаем, что $\left|\mu_{k}\right|>\max (1,4 d) \forall k \in \mathbb{N}$.

Очевидно, для любого $\varepsilon>0$ существуют положительные постоянные $A_{\varepsilon}, B_{\varepsilon}$ такие, что вне множества $\bigcup_{k=1}^{\infty} D\left(\mu_{k}, d\right)$

$$
\begin{aligned}
& |Y(z)| \leqslant A_{\varepsilon} \exp (\varepsilon w(|z|)), \\
& |Y(z)| \geqslant B_{\varepsilon} \exp (-\varepsilon w(|z|)) .
\end{aligned}
$$

Применяя принцип максимума к функции $Y$ в кружках $D\left(\mu_{k}, d\right), k \in \mathbb{N}$, и пользуясь неравенством (2), распространяем оценку вида (4) на всю комплексную плоскость.

Для произвольных $\nu>0, b \in\left(0, d_{2}\right)$ пусть $\Omega(b, \nu)=\bigcup_{k=1}^{\infty} D\left(\mu_{k}, b\left|\mu_{k}\right|^{-\nu}\right)$. Оценим $|Y(z)|$ снизу вне множества $\Omega(b, \nu)$. С этой целью для каждого $k \in \mathbb{N}$ рассмотрим функцию $Y_{k}(z)=\left(z-\mu_{k}\right) Y^{-1}(z), z \in \overline{D\left(\mu_{k}, d\right)}$. Функция $Y_{k}(z)$ аналитична в $\overline{D\left(\mu_{k}, d\right)}$ и не имеет там нулей. По принципу максимума модуля $\left|Y_{k}(z)\right| \leqslant d\left|Y\left(z_{0}\right)\right|^{-1}, z \in D\left(\mu_{k}, d\right)$, где $z_{0}$ - точка минимума $|Y|$ на окружности $\left\{z \in \mathbb{C}:\left|z-\mu_{k}\right|=d\right\}$. Отсюда, пользуясь неравенствами (5) и (2), получаем, что для любого $\varepsilon>0$ найдется постоянная $\gamma_{\varepsilon}>0$, не зависящая от $k$, такая, что $\left|Y_{k}(z)\right| \leqslant \gamma_{\varepsilon} \exp (\varepsilon w(|z|)), z \in D\left(\mu_{k}, d\right)$. Следовательно,

$$
|Y(z)| \geqslant \gamma_{\varepsilon}^{-1}\left|z-\mu_{k}\right| \exp (-\varepsilon w(|z|)), \quad z \in D\left(\mu_{k}, d\right) .
$$

Таким образом, для $z \in D\left(\mu_{k}, d\right) \backslash D\left(\mu_{k}, b\left|\mu_{k}\right|^{-\nu}\right)$

$$
|Y(z)| \geqslant \gamma_{\varepsilon}^{-1} b\left|\mu_{k}\right|^{-\nu} \exp (-\varepsilon w(|z|)) \text {. }
$$

Так как для $z \in D\left(\mu_{k}, d\right)\left|\mu_{k}\right| \leqslant 4|z| / 3$, из вьшеприведенного неравенства следует, что каково бы ни было $\varepsilon>0$ для $z \in D\left(\mu_{k}, d\right) \backslash D\left(\mu_{k}, b\left|\mu_{k}\right|^{-\nu}\right)$ справедлива оценка

$$
|Y(z)| \geqslant \gamma_{\varepsilon}^{-1} b\left(\frac{3}{4}\right)^{\nu} \exp (-\varepsilon w(|z|)-\nu \ln |z|) .
$$

Из (5), (6) с учетом того, что $\ln r=o(w(r))$ при $r \rightarrow+\infty$, следует, что для любого $\varepsilon>0$ найдется постоянная $m_{\varepsilon}>0$ (зависящая от $b, \nu$ ) такая, что

$$
|Y(z)| \geqslant m_{\varepsilon} \exp (-\varepsilon w(|z|)), \quad z \notin \Omega(b, \nu) .
$$

Таким образом, для целой функции $Y(z)$ вьполнены условие L1 и при $\nu \geqslant \alpha-1$ условие L2. 
4. Вспомогательные утверждения. Отметим вначале, что условия V1, V2 накладьвают определенные ограничения на рост функции $v$. Например, из V1 следует неравенство

$$
v(x)>\frac{a x \ln x}{4 \ln 2}+\left(\frac{v(1)}{2}-\frac{a}{4}-|b|\right) x-b, \quad x \geqslant 2 .
$$

Из условия V2 следует, что для любых $\varepsilon \in\left(0, \varepsilon_{0}\right), x, y \geqslant 1$

$$
v(x+y) \leqslant v(x)+\varepsilon x+v(y)+a_{\varepsilon} y+b_{\varepsilon} .
$$

В частности, при $x \geqslant 1, \varepsilon \in\left(0, \varepsilon_{0}\right)$ имеем $v(2 x) \leqslant 2 v(x)+\left(a_{\varepsilon}+\varepsilon\right) x+b_{\varepsilon}$, а из этого неравенства легко следует, что

$$
v(x) \leqslant\left(2 v(1)+a_{\varepsilon}+2 b_{\varepsilon}+\varepsilon\right) x+\frac{\left(a_{\varepsilon}+\varepsilon\right) x \ln x}{\ln 2}-b_{\varepsilon}, \quad x \geqslant 1 .
$$

Введем функцию

$$
h(s)=\varliminf_{x \rightarrow+\infty}\left(\frac{v(x)}{x}-\frac{v(s x)}{s x}\right), \quad s>0 .
$$

Отметим некоторые нужные для дальнейшего свойства этой функции.

ЛЕмма 2. Функиия $h(s)$ обладает свойствами:

1) функиия $h(s)$ принимает конечные значения на положительной полуоси;

2) $h(s) \geqslant 0$ для $s \in(0,1) u h(s) \leqslant 0$ для $s \geqslant 1$;

3) $h(s)$ - невозрастающая функиия на положсительной полуоси;

4) $\lim _{x \rightarrow 0, x>0} h(x)=+\infty$;

5) $\lim _{x \rightarrow 1, x>1} h(x)=0$.

ДоказАтЕльство. Докажем вначале первое свойство. Пусть $s>1$. Тогда для некоторого $N \in \mathbb{N} s \in(N, N+1]$. Из неравенства (7) вьводим, что для любых $x \geqslant(s-N)^{-1}, \varepsilon \in\left(0, \varepsilon_{0}\right)$

$$
v(s x) \leqslant N v(x)+\varepsilon N x+\frac{a_{\varepsilon} N x(2 s-N-1)}{2}+N b_{\varepsilon}+v((s-N) x) .
$$

Отсюда, учитьвая, что $v(t x) \leqslant t v(x)$ для всех $t \in[0,1], x \geqslant 0$, получаем, что для любых $N \in \mathbb{N}, s \in(N, N+1], x \geqslant(s-N)^{-1}, \varepsilon \in\left(0, \varepsilon_{0}\right)$ справедливо неравенство

$$
v(s x) \leqslant s v(x)+\left(\varepsilon+\frac{a_{\varepsilon}(2 s-N-1)}{2}\right) s x+b_{\varepsilon} s .
$$

В частности, найдется постоянная $K_{s}>0$ такая, что для всех $x \geqslant 0$ будет вьполнено неравенство

$$
v(s x) \leqslant s v(x)+\left(\varepsilon+\frac{a_{\varepsilon} s}{2}\right) s x+b_{\varepsilon} s+K_{s} .
$$

Воспользовавшись неравенством $(10)$, получаем $h(s) \geqslant-\varepsilon-0,5 a_{\varepsilon} s$ для всех $s>1$, $\varepsilon \in\left(0, \varepsilon_{0}\right)$. Для $s \in(0,1)$

$$
h(s)=\varliminf_{x \rightarrow+\infty}\left(\frac{v\left(s^{-1} x\right)}{s^{-1} x}-\frac{v(x)}{x}\right),
$$


и поэтому, снова пользуясь $(10)$, имеем $h(s) \leqslant \varepsilon+0,5 a_{\varepsilon} s^{-1}$.

Поскольку из определения $h(1)=0$, первое свойство полностью доказано.

Второе и третье свойства следуют из того, что благодаря вьпуклости $v$ и условию $v(0)=0$ функция $v(x) / x$ не убьвает на положительной полуоси.

Из условия V1 имеем для любых $x \geqslant 1, n \in \mathbb{N} v\left(2^{n} x\right) \geqslant 2^{n} v(x)+a n 2^{n-1} x+b\left(2^{n}-1\right)$. Следовательно, $h\left(2^{-n}\right) \geqslant a n / 2$. Отсюда и из невозрастания функции $h$ получаем третье свойство функции $h$.

Покажем, что предел функции $h$ справа в точке 1 равен 0 . Пусть $\varepsilon \in\left(0, \varepsilon_{0}\right)$ произвольно, $s \in(1,2)$. Воспользовавшись $(9)$, имеем $h(s) \geqslant-\varepsilon-a_{\varepsilon}(s-1)$. Таким образом, если $0<s-1<\min \left(1, \varepsilon a_{\varepsilon}^{-1}\right)$, то $-2 \varepsilon<h(s)-h(1) \leqslant 0$. Отсюда следует, что функция $h(s)$ непрерьвна справа в точке 1 .

Лемма 2 доказана.

ЛЕмма 3. Справедливо равенство

$$
h(s)=\underset{k \rightarrow+\infty}{\lim _{\rightarrow}}\left(\frac{v(k)}{k}-\frac{v([s k]+1)}{s k}\right), \quad s>0 .
$$

ДокаЗАТЕЛЬСтво. Пусть $s>0$. Для $x \in[k, k+1), k \in \mathbb{N}$, имеем

$$
\begin{gathered}
\frac{v(x)}{x}-\frac{v(s x)}{s x} \geqslant \frac{v(k)}{k}-\frac{v(s k+s)}{s k}=\frac{v(k)}{k}-\frac{v([s k]+1)}{s k}+\frac{v([s k]+1)-v(s k+s)}{s k}, \\
\frac{v(x)}{x}-\frac{v(s x)}{s x} \leqslant \frac{v(k+1)}{k+1}-\frac{v(s k)}{s k} \leqslant \\
\frac{v(k)}{k}-\frac{v([s k]+1)}{s k}+\frac{v(s k+1)-v(s k)}{s k} \\
+\frac{v(k+1)-v(k)}{k+1}-\frac{v(k)}{k(k+1)} .
\end{gathered}
$$

Учитывая первое условие на $v$ и неравенство (8), получаем из этих оценок искомое равенство. Лемма доказана.

Положим $l(s)=\exp (h(s)), s>0$.

Лемма 4. Для любых $s>0, \delta \in(0,1)$ существует постоянная $Q(s, \delta) \geqslant 0$ такая, что для всех $r \geqslant 0$

$$
s w(r) \leqslant w\left(\frac{r}{l(s)(1-\delta)}\right)+Q(s, \delta) .
$$

ДокАЗАТЕЛЬСтво. Пользуясь леммой 3 , для любых $s>0, \delta \in(0,1)$ найдем постоянную $A(s, \delta)>0$ такую, что для всех $k \in \mathbb{Z}$

$$
s v(k)>v([s k]+1)+s k(h(s)+\ln (1-\delta))-A(s, \delta) .
$$

Поэтому для $s \in(0,1), r>1$

$$
\begin{aligned}
s w(r) & =\sup _{k \in \mathbb{Z}_{+}}(s k \ln r-s v(k)) \\
& \leqslant \sup _{k \in \mathbb{Z}_{+}}(([s k]+1) \ln r-v([s k]+1)-([s k]+1)(h(s)+\ln (1-\delta)))+h(s)+A(s, \delta) \\
& \leqslant \sup _{N \in \mathbb{Z}_{+}}\left(N \ln \frac{r}{l(s)(1-\delta)}-v(N)\right)+h(s)+A(s, \delta) \\
& =w\left(\frac{r}{l(s)(1-\delta)}\right)+h(s)+A(s, \delta) .
\end{aligned}
$$


Аналогично, для $s \geqslant 1, r>1$

$$
s w(r) \leqslant w\left(\frac{r}{l(s)(1-\delta)}\right)+A(s, \delta) .
$$

Следовательно, при $r>1$ утверждение леммы справедливо. Выбирая надлежащим образом постоянную $Q(s, \delta) \geqslant 0$, добьемся выполнения неравенства для всех $r \geqslant 0$.

Лемма 4 доказана.

Лемма 5. Для любого $n \in \mathbb{N}$ существуют числа $\varepsilon>0, q \geqslant 0$ такие, что

$$
w_{n}(|z|)+\varepsilon w(|z|) \leqslant w_{n+1}(|z|)+q, \quad z \in \mathbb{C} .
$$

ДокАЗАТЕЛЬСТво. Пусть $n \in \mathbb{N}$. Выберем числа $\mu, \delta$ из интервала $(0,1)$ настолько малыми, что $l(\mu)>2\left(\sigma+\varepsilon_{n}\right)$ и $\left(\sigma+\varepsilon_{n}\right)(1-\delta) l(1+\mu)>\sigma+\varepsilon_{n+1}$. В силу свойств 4$)$ и 5$)$ функции $h$ это возможно. Пользуясь леммой 4 и обозначениями из этой леммы, имеем при любом $z \in \mathbb{C}$

$$
\begin{aligned}
w_{n}(|z|)+\mu^{2} w(|z|) & \leqslant w_{n}(|z|)+\mu w\left(\frac{2|z|}{l(\mu)}\right)+\mu Q\left(\mu, 2^{-1}\right) \\
& \leqslant(1+\mu) w_{n}(|z|)+\mu Q\left(\mu, 2^{-1}\right) \\
& \leqslant w\left(\frac{|z|}{\left(\sigma+\varepsilon_{n}\right)(1-\delta) l(1+\mu)}\right)+\mu Q\left(\mu, 2^{-1}\right)+Q(1+\mu, \delta) \\
& \leqslant w_{n+1}(|z|)+q,
\end{aligned}
$$

где $q=\mu Q\left(\mu, 2^{-1}\right)+Q(1+\mu, \delta)$. Положим теперь $\varepsilon=\mu^{2}$.

Лемма доказана.

Учитывая, что $\ln (1+r)=o(w(r))$ при $r \rightarrow+\infty$, из этой леммы получаем

СледСтвиЕ. Для любых $A>0, n \in \mathbb{N}$ существует постоянная $Q \geqslant 0$ такая, чmo

$$
w_{n}(|z|)+A \ln (1+|z|) \leqslant w_{n+1}(|z|)+Q, \quad z \in \mathbb{C} .
$$

ЗАмЕчаниЕ. Утверждение, содержашееся в следствии из леммы 5 , справедливо в предположении, что $v$ лишь принадлежит $V$ (см. [2]).

Лемма 6. Если для $F \in P$ отношение $F / L$ - иелая функиия, то $F / L \in P$.

ДокАЗАТЕльСтво. Так как $F \in P$, то при некоторых $m \in \mathbb{N}, c>0$

$$
|F(z)| \leqslant c \exp \left(\psi(\operatorname{Im} z)+w_{m}(|z|)\right), \quad z \in \mathbb{C} .
$$

Согласно лемме 5 найдутся числа $\varepsilon>0, q \geqslant 0$ такие, что

$$
w_{m}(|z|)+2 \varepsilon w(|z|) \leqslant w_{m+1}(|z|)+q, \quad z \in \mathbb{C} .
$$

Считаем, что $\varepsilon$ настолько мало, что $\left(\sigma+\varepsilon_{m+1}\right)(1+\varepsilon)^{-1}>\sigma+\varepsilon_{m+2}$. 
Если $z \notin S$, то, пользуясь (3), (11), (12), получаем

$$
\left|\frac{F(z)}{L(z)}\right| \leqslant c c_{\varepsilon}^{-1} \exp \left(\psi(\operatorname{Im} z)+w_{m+1}(|z|)+q\right) .
$$

Пусть $z \in S$. Тогда $z \in D\left(\lambda_{n}, r_{n}\right)$ при некотором $n \in \mathbb{N}$. Применяя принцип максимума модуля к функции $F / L$ в круге $D\left(\lambda_{n}, r_{n}\right)$ и пользуясь оценками $(3),(11)$, получаем

$$
\left|\frac{F(z)}{L(z)}\right| \leqslant c c_{\varepsilon}^{-1} \exp \left(\psi\left(\operatorname{Im} t_{0}\right)+w_{m}\left(\left|t_{0}\right|\right)+\varepsilon w\left(\left|t_{0}\right|\right)\right),
$$

где $t_{0}$ - точка максимума $|F(t) / L(t)|$ на окружности $\left\{t \in \mathbb{C}:\left|t-\lambda_{n}\right|=r_{n}\right\}$. Оценим сверху $\psi\left(\operatorname{Im} t_{0}\right), w_{m}\left(\left|t_{0}\right|\right)$ и $w\left(\left|t_{0}\right|\right)$. Отметим вначале, что ввиду неравенства $w(r) \leqslant C_{v} r$ при $r \geqslant 0$ и условия L2 на функцию $L$ имеем $\lim _{j \rightarrow \infty}\left(r_{j} /\left|\lambda_{j}\right|\right)=0$. Поэтому можно найти номер $N$ такой, что для всех $j \geqslant N, t_{1}, t_{2} \in \overline{D\left(\lambda_{j}, r_{j}\right)}=\left\{z \in \mathbb{C}:\left|z-\lambda_{j}\right| \leqslant r_{j}\right\}$ будет справедливо неравенство $\left|t_{1}\right| /\left|t_{2}\right|<1+\varepsilon$. Следовательно, если $j \geqslant N$, то для любых $t_{1}, t_{2} \in \overline{D\left(\lambda_{j}, r_{j}\right)} w_{m}\left(\left|t_{1}\right|\right)<w_{m}\left((1+\varepsilon)\left|t_{2}\right|\right)$ и $w\left(\left|t_{1}\right|\right) \leq w\left((1+\varepsilon)\left|t_{2}\right|\right)$. Подберем постоянную $K_{1}$ так, чтобы для любых $j \in \mathbb{N}, t_{1}, t_{2} \in \overline{D\left(\lambda_{j}, r_{j}\right)}$ вьполнялись неравенства $w_{m}\left(\left|t_{1}\right|\right)<w_{m}\left((1+\varepsilon)\left|t_{2}\right|\right)+K_{1}$ и $w\left(\left|t_{1}\right|\right)<w\left((1+\varepsilon)\left|t_{2}\right|\right)+K_{1}$. Далее, воспользовавшись неравенством (1) и условием $\mathrm{S} 2$ на исключительные кружки, можно найти постоянную $K_{2}>0$, не зависящую от $j$, такую, что для любых $t_{1}, t_{2} \in \bar{D}\left(\lambda_{j}, r_{j}\right)$ $\left|\psi\left(\operatorname{Im} t_{1}\right)-\psi\left(\operatorname{Im} t_{2}\right)\right| \leqslant \varepsilon w\left(\left|\lambda_{j}\right|\right)+K_{2}$. Таким образом,

$$
\begin{aligned}
\psi\left(\operatorname{Im} t_{0}\right) & \leqslant \psi(\operatorname{Im} z)+\varepsilon w\left(\left|\lambda_{n}\right|\right)+K_{2} \\
& <\psi(\operatorname{Im} z)+\varepsilon w((1+\varepsilon)|z|)+\varepsilon K_{1}+K_{2}
\end{aligned}
$$

Пользуясь полученными оценками и выбором $\varepsilon$, имеем из (14) для $z \in S$

$$
\left|\frac{F(z)}{L(z)}\right| \leqslant c c_{\varepsilon}^{-1} \exp \left(\psi(\operatorname{Im} z)+w_{m+2}(|z|)+K_{1}+2 \varepsilon K_{1}+K_{2}+q\right) .
$$

Из оценок (13) и (15) следует, что $F / L \in P$. Лемма доказана.

Обозначим через $Z(L)$ множество нулей функции $L$.

Лемма 7. Система $W=\{\exp (-i x z)\}_{z \in \mathbb{C} \backslash Z(L)}$ полна в $G$.

ДокАЗАТЕЛЬСтво. Чтобы доказать лемму, нужно показать, что если для произвольного функционала $T \in G^{*} T(f)=0 \forall f \in W$, то $T(f)=0 \forall f \in G$. Поскольку $\widehat{T}(z)=0, z \in \mathbb{C} \backslash Z(L)$, то по теореме единственности $\widehat{T}=0$. Тогда по теореме $\mathrm{A} T=0$. Лемма доказана.

5. Доказательство теоремы. Покажем вначале, что линейньй оператор $\mathscr{L}(f)=$ $\sum_{k=0}^{\infty} d_{k} i^{k} f^{(k)}, f \in G$, действует из $G$ в $G$ непрерывно. Пользуясь леммой 4 и свойством 4) функции $h$, из оценки на $|L|$ получаем, что для любого $\varepsilon>0$ существует постоянная $M_{\varepsilon}>0$ такая,что для любого $z \in \mathbb{C}|L(z)| \leqslant M_{\varepsilon} \exp (w(\varepsilon|z|))$. Из этой оценки, равенства (см. [3, с. 24])

$$
\inf _{r>0} \frac{\exp (w(r))}{r^{k}}=\exp (-v(k)), \quad k \in \mathbb{Z}_{+},
$$


и неравенства Коши для коэффициентов степенного ряда получаем, что для любого $\varepsilon>0$ справедлива оценка

$$
\left|d_{k}\right| \leqslant M_{\varepsilon} \varepsilon^{k} \exp (-v(k)), \quad k \in \mathbb{Z}_{+} .
$$

Пользуясь последней оценкой и условием $\mathrm{V} 2$, имеем при любых $\varepsilon>0, \delta \in\left(0, \varepsilon_{0}\right)$, $m \in \mathbb{Z}_{+}, n \in \mathbb{N}, f \in G, x \in \mathbb{R}$

$$
\begin{aligned}
& \sum_{k=0}^{\infty}\left|d_{k}\right|\left|f^{(k+m)}(x)\right| \leqslant M_{\varepsilon} p_{n+1}(f) \theta_{n+1}(x) \sum_{k=0}^{\infty} \varepsilon^{k}\left(\sigma+\varepsilon_{n+1}\right)^{k+m} e^{v(k+m)-v(k)} \\
& \quad \leqslant M_{\varepsilon} e^{v(m)+\delta m+b_{\delta}} p_{n+1}(f) \theta_{n+1}(x)\left(\sigma+\varepsilon_{n+1}\right)^{m} \sum_{k=0}^{\infty} \varepsilon^{k}\left(\sigma+\varepsilon_{n+1}\right)^{k} \exp \left(a_{\delta} k\right) .
\end{aligned}
$$

Выбирая теперь $\delta>0$ настолько маленьким, чтобы вьполнялось неравенство $e^{\delta}(\sigma+$ $\left.\varepsilon_{n+1}\right)<\sigma+\varepsilon_{n}$, а затем $\varepsilon>0$ так, чтобы $\varepsilon\left(\sigma+\varepsilon_{n+1}\right) \exp \left(a_{\delta}\right)$ было меньше 1 , получим оценку

$$
\sum_{k=0}^{\infty}\left|d_{k}\right|\left|f^{(k+m)}(x)\right| \leqslant V_{n} p_{n+1}(f) \theta_{n+1}(x)\left(\sigma+\varepsilon_{n}\right)^{m} e^{v(m)},
$$

где $V_{n}>0$ - некоторая постоянная. Следовательно, $\mathscr{L}(f) \in G$ и справедливо неравенство $p_{n}(\mathscr{L}(f)) \leqslant V_{n} p_{n+1}(f)$. Последнее неравенство означает, что $\mathscr{L}$ действует из $G$ в $G$ непрерьвно.

Линейньй оператор $\mathscr{L}$ определяет сопряженный оператор $\mathscr{L}^{*}: G^{*} \rightarrow G^{*}$ по правилу $(\mathscr{L}(f), T)=\left(f, \mathscr{L}^{*}(T)\right), f \in G, T \in G^{*}$. Так как пространства $G^{*}$ и $P$ топологически изоморфны, то $\mathscr{L}^{*}$ порождает оператор $\widehat{\mathscr{L}}: P \rightarrow P$ по формуле $\widehat{T} \rightarrow \widehat{\mathscr{L}}(T), T \in G^{*}$. Легко видеть, что $\widehat{\mathscr{L}}(F)(z)=F(z) L(z), F \in P$.

Образ произвольного оператора $A$ будем обозначать через $\operatorname{im} A$.

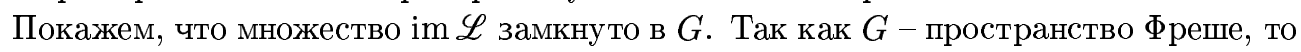
замкнутость $\operatorname{im} \mathscr{L}$ в $G$ равносильна замкнутости $\operatorname{im} \mathscr{L}^{*}$ в $G^{*}$ [8], а последнее имеет место тогда и только тогда, когда множество im $\widehat{\mathscr{L}}$ замкнуто в $P$. Согласно $[1$, теорема 1$]$ im $\widehat{\mathscr{L}}$ замкнуто в $P$ тогда и только тогда, когда $\operatorname{im} \widehat{\mathscr{L}} \cap P_{m}$ замкнуто в $P_{m}$ для любого $m \in \mathbb{N}$.

Пусть $m \in \mathbb{N}$ произвольно. Пусть $F$ принадлежит замыканию $\operatorname{im} \widehat{\mathscr{L}} \cap P_{m}$ в $P_{m}$. Тогда существует последовательность $\left\{F_{n}\right\}_{n=1}^{\infty} \subset \mathrm{im} \hat{\mathscr{L}} \cap P_{m}$, сходящаяся к $F$ в топологии $P_{m}$, т.e. $\forall \varepsilon>0 \exists N(\varepsilon) \in \mathbb{N}: \forall n>N(\varepsilon)$

$$
\left|F(z)-F_{n}(z)\right| \leqslant \varepsilon \exp \left(\psi(\operatorname{Im} z)+w_{m}(|z|)\right), \quad z \in \mathbb{C} .
$$

Если $\zeta_{j}-$ нуль $L$ кратности $s_{j}$, то для $k=0,1, \ldots, s_{j}-1$

$$
\left|F^{(k)}\left(\zeta_{j}\right)\right|=\left|F^{(k)}\left(\zeta_{j}\right)-F_{n}^{(k)}\left(\zeta_{j}\right)\right| \leqslant \frac{k !}{r^{k}} \max _{\left|\zeta-\zeta_{j}\right|=r}\left|F(\zeta)-F_{n}(\zeta)\right|,
$$

где $r=\left(\left|\zeta_{j}\right|+1\right)^{1-\alpha}$. Пользуясь $(16),(1),(2)$ и следствием из леммы 5, получаем при $k=0,1, \ldots, s_{j}-1$

$$
\left|F^{(k)}\left(\zeta_{j}\right)\right| \leqslant \varepsilon l_{k} \exp \left(\psi\left(\operatorname{Im} \zeta_{j}\right)+w_{m+1}\left(\left|\zeta_{j}\right|\right)\right)
$$


где положительная постоянная $l_{k}$ не зависит от $j$. В силу произвольности $\varepsilon>0$ имеем $F^{(k)}\left(\zeta_{j}\right)=0, k=0,1, \ldots, s_{j}-1$. Отсюда следует, что $F / L$-целая функция. По лемме 6 $F / L \in P$. Следовательно, $F \in \operatorname{im} \widehat{L} \cap P_{m}$. Это означает, что множество im $\widehat{\mathscr{L}}$ замкнуто в $P$. Следовательно, множество $\operatorname{im} \mathscr{L}$ замкнуто в $G$.

Покажем теперь, что $\operatorname{im} \mathscr{L}=G$. Рассмотрим уравнение $\mathscr{L}(f)=\exp (-i x z)$, где $z \in \mathbb{C} \backslash Z(L)$. Оно имеет решение $f(z)=\exp (-i x z) / L(z)$. Учитывая лемму 7 , делаем вывод, что множество $\operatorname{im} \mathscr{L}$ плотно в $G$. Из этого и из замкнутости im $\mathscr{L}$ в $G$ следует, что $\operatorname{im} \mathscr{L}=G$.

Теорема доказана.

Автор признателен рецензенту за ценные замечания.

\section{СПИСОК ЦИТИРОВАННОЙ ЛИТЕРАТУРЫ}

[1] Себаштьян-и-Сильва ЖК. О некоторых классах локально выпуклых пространств, важных в приложениях // Математика. 1957. Т. 1. № 1. С. 60-77.

[2] Musin I. Kh. On the Fourier-Laplace transform of functionals on a weighted space of infinitely differentiable functions // E-print funct-an/9911067, 1999.

[3] Мандельбройт С. Примькающие ряды, регуляризация последовательностей, применения. М.: ИЛ, 1955.

[4] Мусин И.Х. О преобразовании Фурье-Лапласа функционалов на весовом пространстве бесконечно дифференцируемых функций // Матем. сб. 2000. Т. 191. № 10. С. 57-86.

[5] Мусин И. Х. Теорема типа Пэли-Винера для весового пространства бесконечно дифференцируемых функций // Изв. РАН. Сер. матем. 2000. Т. 64. №6. С. 181-204.

[6] Напалков В. В. Уравнения свертки в многомерных пространствах. М.: Наука, 1982.

[7] Кривошеев А. С., Напалков В. В. Комплексньй анализ и операторы свертки // УМН. 1992. T. 47. №6. C. 3-58.

[8] Дьедонне ЖК., Шварц Л. Двойственность в пространствах $(F)$ и $(L F) / /$ Математика. 1958. T. 2. № 2. C. 77-107.

[9] Ehrenpreis L. Solution of some problems of division. IV. // Amer. J. Math. 1960. V. 82. P. 522-588.

[10] Malgrange B. Éxistence et approximation des solutions des équations aux derivées partielles et des équations de convolution // Ann. Inst. Fourier (Grenoble). 1955-56. V. 6. P. 271-355.

[11] Meise R., Taylor B. A., Vogt D. Equivalence of slowly decreasing conditions and local Fourier expansions // Indiana Univ. Math. J. 1987. V. 36. P. 729-756.

[12] Momm S. Closed ideals in nonradial Hörmander algebras // Arch. Math. 1992. V. 58. P. 47-55.

[13] Braun R. W., Meise R., Vogt D. Characterization of the linear partial differential operators with constant coefficients which are surjective on non-quasianalytic classes of Roumieu type on $\mathbb{R}^{N}$ // Math. Nachr. 1994. V. 168. P. 19-54.

[14] Bjorck G. Linear partial differential operators and generalized distributions // Ark. Mat. 1965. V. 6. P. 351-407.

[15] Braun R. W., Meise R., Taylor B. A. Ultradifferentiable functions and Fourier analysis // Results in Math. 1990. V. 17. P. 205-237.

[16] Коробейник И.Ф. О бесконечно дифференцируемых решениях линейного дифференциального уравнения бесконечного порядка // Сиб. матем. ж. 1965. Т. 6. № 3. С. 516-527.

[17] Фихтенгольц Г. М. Курс дифференциального и интегрального исчисления. Т. ІІ. М.-Л.: ГИТТЛ, 1948.

[18] Юлмухаметов Р. С. Аппроксимация субгармонических функций // Analysis Math. 1985. V. 11. № 3. P. 257-282.

Институт математики с ВЦ Уфимского НЦ РАН, г. Уфа

Поступило

E-mail: musin@imath.rb.ru

11.01.2000

Исправленный вариант

03.01.2001 